\title{
Tandem Interface and Bulk Li-lon Transport in a Hybrid Solid Electrolyte with Microsized Active Filler
}

Ming Liu, ${ }^{\dagger}$ Zhu Cheng, ${ }^{\S}$ Swapna Ganapathy, ${ }^{\dagger}$ Chao Wang, ${ }^{\dagger}$ Lucas A. Haverkate, ${ }^{\ddagger}$ Michał Tułodziecki, ${ }^{\dagger}$ Sandeep Unnikrishnan, and Marnix Wagemaker*, ${ }^{+}$

${ }^{\dagger}$ Section Storage of Electrochemical Energy, Radiation Science and Technology, Faculty of Applied Sciences, Delft University of Technology, 2629 JB Delft, The Netherlands

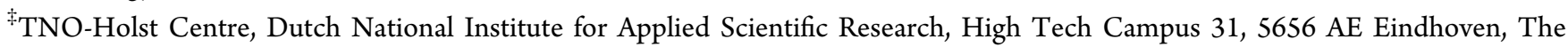
Netherlands

${ }^{\S}$ Center of Energy Storage Materials \& Technology, College of Engineering and Applied Sciences, National Laboratory of Solid State Microstructures, Collaborative Innovation Center of Advanced Microstructures, Nanjing University, Nanjing 210008, China

Supporting Information

ABSTRACT: In common hybrid solid electrolytes (HSEs), either the ionic conductivity of the polymer electrolyte is enhanced by the presence of a nanosized inorganic filler, which effectively decrease the glass-transition temperature, or the polymer solid electrolyte acts mostly as a flexible host for the inorganic solid electrolyte, the latter providing the conductivity. Here a true HSE is developed that makes optimal use of the high conductivity of the inorganic solid electrolyte and the flexibility of the polymer matrix. It is demonstrated that the LAGP $\left(\mathrm{Li}_{1.5} \mathrm{Al}_{0.5} \mathrm{Ge}_{1.5}\left(\mathrm{PO}_{4}\right)_{3}\right)$ participates in the overall conductivity and that the interface environment between the poly(ethylene oxide) (PEO) and LAGP plays a key role in utilizing the high conductivity of the LAGP. This HSE demonstrates promising cycling versus $\mathrm{Li}$-metal anodes and in a full Li-metal solid-state battery. This strategy offers a promising route for the development of Li-metal solid-state batteries, aiming for safe and reversible high-energy-density batteries. (a)

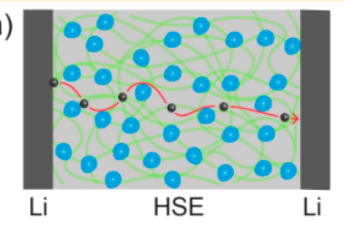

(b)

(c)

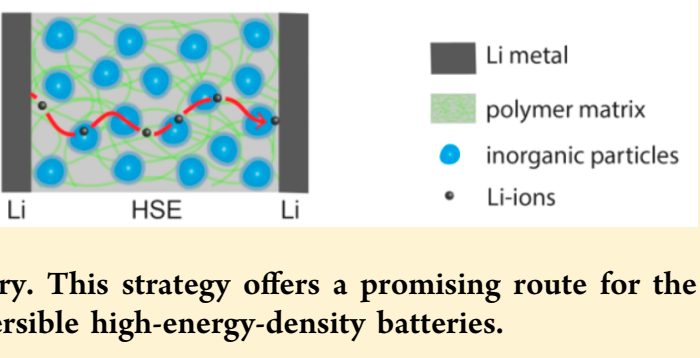

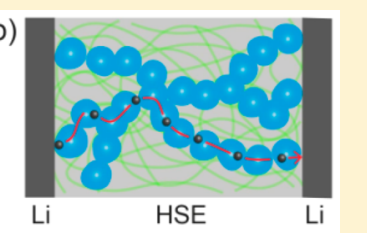

Li metal
polymer matrix
- inorganic particles
- Li-ions
1 remendous effort is being invested in exploring nextgeneration rechargeable batteries, which possess an energy density that exceeds current Li-ion battery (LIB) technology. This is essential in order to expand the applicability of portable devices and electric vehicles. The development of state-of-the-art LIBs is in particular restricted by the limited lithium ( $\mathrm{Li}$ ) storage capacity of its graphitic carbon anodes. ${ }^{1-3}$ In terms of high specific capacity (3860 $\left.\mathrm{mAh}^{-1}\right)$ and low reduction potential ( $-3.040 \mathrm{~V}$ vs SHE), Limetal is considered the "Holy Grail" of Li-battery anodes, making it a topic of intensive study. ${ }^{3,4}$ In recent years, progress in the development of high-capacity cathodes such as sulfur-, oxygen-, and Li-rich cathodes, has also led to the revival of $\mathrm{Li}$ metal anode research, currently making it a hot topic. ${ }^{5-8}$ Unfortunately, the thick solid electrolyte interface (SEI), formed due to severe reactivity with the electrolyte, and related growth of lithium dendrites result in safety concerns that have hindered the capitalization of Li-metal-based batteries (LMBs). 3,9 Many strategies to alleviate lithium dendrite growth have been developed, including the use of electrolyte

. 

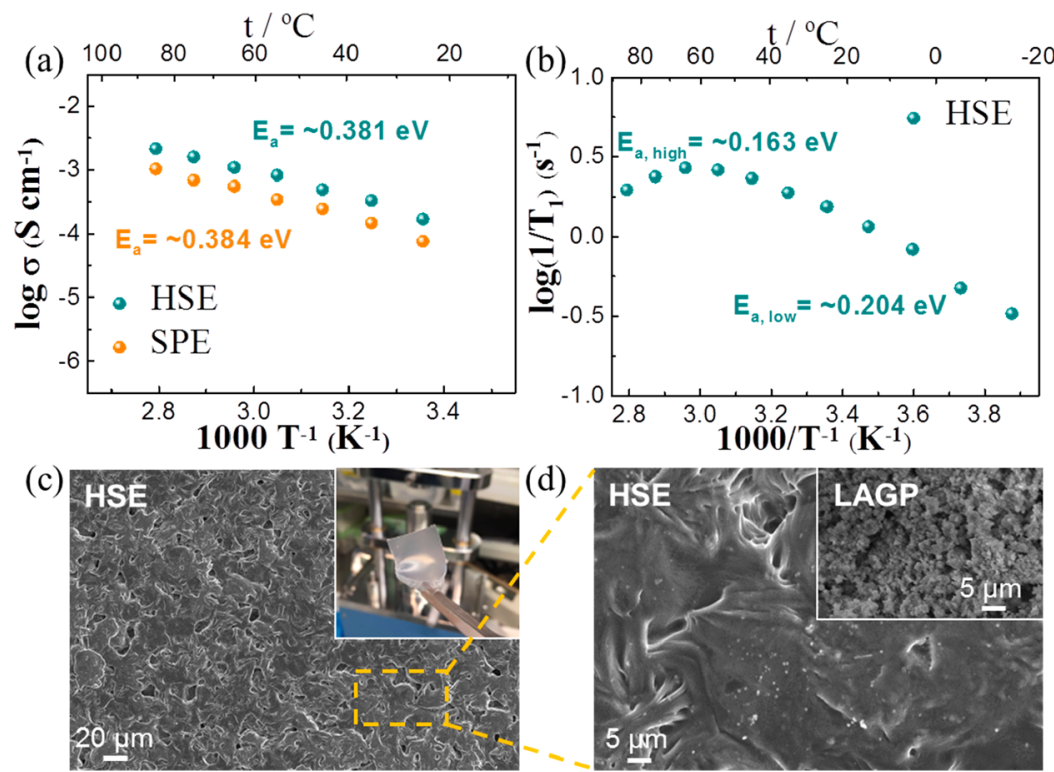

Figure 1. Characterization of the HSE. (a) Ionic conductivity determined by impedance spectroscopy measurements of the HSE and SPE at different temperatures. (b) $T_{1}$ determined by ${ }^{7} \mathrm{Li}$ NMR SLR experiments at different temperatures for the HSE. (c,d) SEM measurements showing the morphology of the HSE and the LAGP particles. The optical image of the HSE film is shown in the inset of (c).

Among the SPEs, the most well-known and intensively studied family are polyether-based, i.e., poly(ethylene oxide) (PEO)based polymers, discovered by Wright in 1973 and first applied in a lithium battery by Armand in $1978 .^{25}$ Because they can directly complex with lithium salts and function as polymer electrolytes without the addition of a liquid plasticizer, these PEO-based electrolytes have attracted a great deal of attention in previous decades. ${ }^{23,26}$ The disadvantage of PEO-based electrolytes is their relatively low room-temperature conductivity $\left(10^{-8}-10^{-6} \mathrm{~S} \mathrm{~cm}^{-1}\right)$, which is ascribed to the semicrystalline nature of PEO $\left(T_{\mathrm{m}}=65{ }^{\circ} \mathrm{C}\right)$, which lowers the PEO chain mobility. Consequentially, existing PEO-based LMBs can work only at elevated temperatures $\left(>60{ }^{\circ} \mathrm{C}\right) .^{23,27}$ This has spawned intensive research toward the development of PEO-based SPEs with improved ionic transport properties while retaining their superior mechanical and process properties. $^{28,29}$ One established strategy is to introduce an inorganic filler in the polymer matrix, thus constructing an $\mathrm{HSE}^{30-37}$ Early work used nanosized inactive fillers, such as $\mathrm{SiO}_{2}, \mathrm{Al}_{2} \mathrm{O}_{3}$, and $\mathrm{TiO}_{2}$, which suppress the crystallization of the PEO chains. This facilitates polymer segmental motion, thereby promoting ionic conduction (percolation effect). ${ }^{30,38}$ Recently, $\mathrm{Hu}$ et al. ${ }^{39}$ traced the Li-ion pathways in an HSE comprising nanosized $\mathrm{Li}_{10} \mathrm{GeP}_{2} \mathrm{~S}_{12}$ (LGPS) particles embedded in a PEO matrix with solid-state nuclear magnetic resonance (ssNMR) spectroscopy. In that case, Li-ion transport was found to dominantly place through the LGPS-PEO interfaces that percolate through the membrane and not through the bulk of the inorganic or polymer phases. Zhang et al. ${ }^{40}$ proposed a flexible anion-immobilized ceramic-polymer composite electrolyte with $40 \%$ microsized $(8.7 \mu \mathrm{m})$ active LLZO filler particles in a PEO matrix. An improved resistance to dendrite penetration was demonstrated, and it was shown that the conductivity occurred via a network of LLZO particles embedded in the PEO matrix. In this case, the PEO matrix is inactive in the Li-transport mechanism and acts primarily as a flexible host. This role of the microsized active filler was also elucidated by $\mathrm{Hu}$ et al. ${ }^{33,34}$ with ssNMR spectroscopy. It was shown that $\mathrm{Li}$ ions favor Li-ion diffusion pathways through the LLZO ceramic phase (50 wt \%) instead of diffusing through the PEO-LLZO interface or the PEO matrix. Between these extremes, i.e., a small amount of nanosized filler (both active and inactive) versus a large amount of active microsized filler, there are possibilities to explore the transport mechanism and achieve improved performance by tuning the HSE composition, where in particular interfacial phenomena can be expected to play a key role.

Here we report the development of a room-temperature solid-state LMB with an HSE, consisting of a PEO matrix with succinonitrile $(\mathrm{SN})$ as an additive and a small amount of microsized $\mathrm{Li}_{1+x} \mathrm{Al}_{x} \mathrm{Ge}_{2-x}\left(\mathrm{PO}_{4}\right)_{3}$ (LAGP) inorganic filler. The Li-ion transport mechanism through the HSE is characterized by ssNMR. It is found that with the addition of $\mathrm{SN}$ conductivity through the LAGP is activated through the interface with the polymer matrix. This allows reduction of the number of inorganic particles added to the electrolyte system, making the HSE more flexible and cheaper while also improving the conductivity of the HSE, thus enabling roomtemperature operation. Through this new approach, optimal use of the high conductivity of the inorganic solid electrolyte and the flexibility of the polymer matrix is achieved. The proposed HSE concept provides new directions and possibilities in the design of HSEs for room-temperature LMBs.

A pure PEO-based electrolyte does not provide sufficient $\mathrm{Li}$ ion conductivity to enable a room-temperature solid-state LMB. Interestingly, Fan et al. ${ }^{41}$ introduced a plastic crystal, SN, into PEO-based polymer electrolytes, resulting in ultrahigh ionic conductivity $\left(>10^{-3} \mathrm{~S} / \mathrm{cm}\right)$. However, in practice, the poor mechanical strength and processability of the pure $\mathrm{PEO} /$ $\mathrm{SN} / \mathrm{Li}$ salt mixture pose a challenge, which may be resolved by the introduction of inorganic fillers in these polymer electrolytes. Intensive research efforts have led to the discovery of several families of excellent inorganic solid Li-ion conductors including the sulfides $\left(\mathrm{Li}_{2} \mathrm{~S}-\mathrm{P}_{2} \mathrm{~S}_{5}, \mathrm{Li}_{2} \mathrm{~S}-\mathrm{SiS}_{2}\right.$, $\left.\mathrm{Li}_{2} \mathrm{~S}-\mathrm{GeS}_{2}\right)$, oxides $\left(\mathrm{Li}_{7} \mathrm{La}_{3} \mathrm{Zr}_{2} \mathrm{O}_{12}\right.$ and $\left.\mathrm{Li}_{3 x} \mathrm{La}_{(2 / 3)-3 x} \mathrm{TiO}_{3}\right)$ 

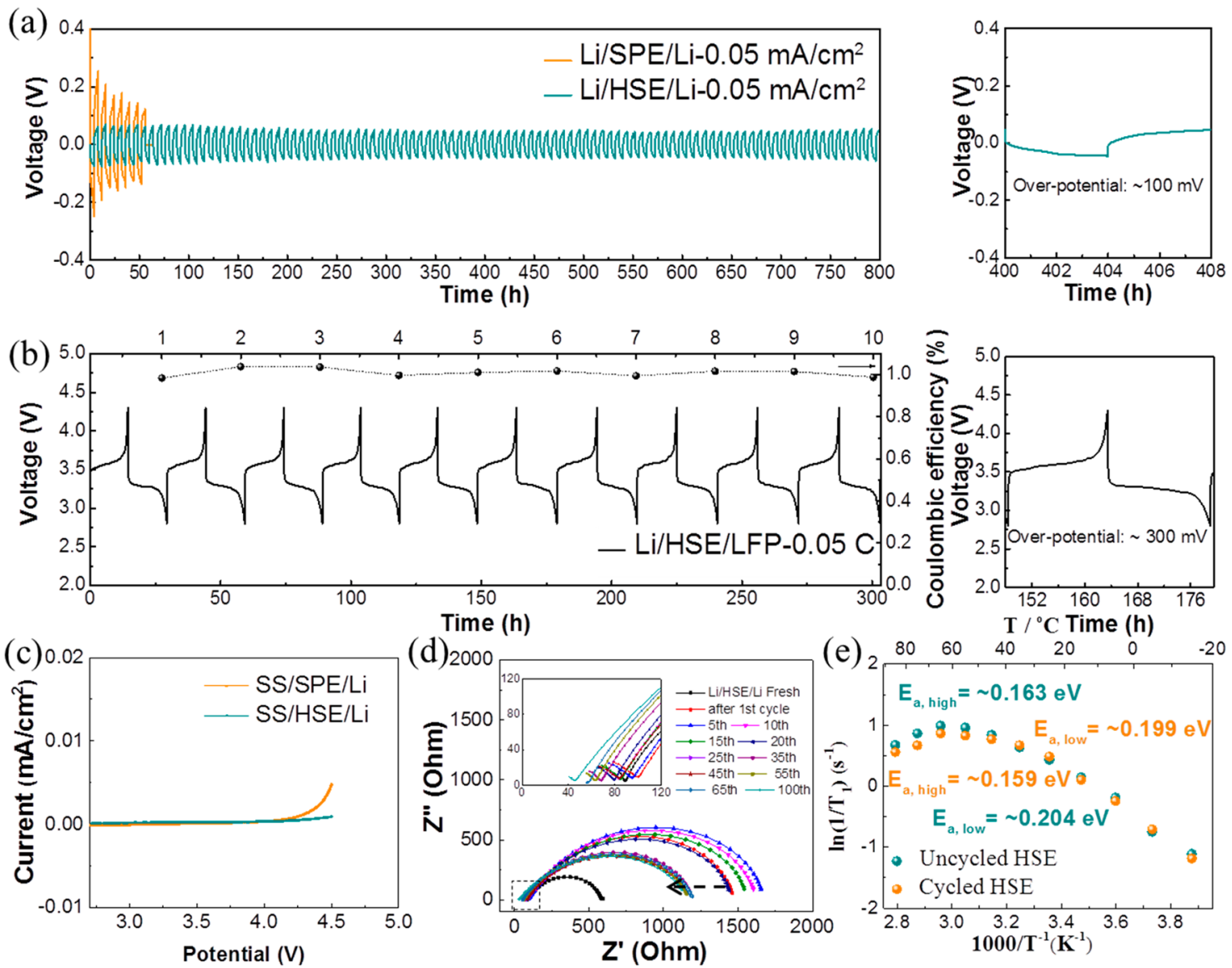

Figure 2. Application of the HSE in a LMB. Electrochemical performance of (a) Li/SPE/Li, Li/HSE/Li, and (b) LFP/HSE/Li batteries at $0.05 \mathrm{~mA} / \mathrm{cm}^{2}$ and $0.05 \mathrm{C}$. The expanded scale is shown in the right panel. (c) LSV of the Li/SPE/SS and Li/HSE/SS half-cells from opencircuit voltage (OCV) to $4.5 \mathrm{~V}$ at a scan rate of $0.1 \mathrm{mV} \mathrm{s}{ }^{-1}$, using stainless steel (SS) as a working electrode and Li as the counter and reference electrodes. (d) Evolution of the EIS of the Li/HSE/Li battery upon cycling at $0.05 \mathrm{~mA} / \mathrm{cm}^{2}$. (e) $T_{1}$ determined by ${ }^{7} \mathrm{Li}$ NMR SLR experiments at different temperatures for the pristine HSE and HSE after 10 cycles in a $\mathrm{Li} / \mathrm{HSE} / \mathrm{Li}$ battery.

and phosphates ( $\mathrm{LiPON}, \mathrm{Li}_{1+x} \mathrm{Al}_{x} \mathrm{Ge}_{2-x}\left(\mathrm{PO}_{4}\right)_{3}$, and $\left.\mathrm{Li}_{1+x} \mathrm{Ti}_{2-x} \mathrm{Al}_{x}\left(\mathrm{PO}_{4}\right)_{3}\right) .{ }^{42}$ Among them, the $\mathrm{Li}_{1+x} \mathrm{Al}_{x} \mathrm{Ge}_{2-x}\left(\mathrm{PO}_{4}\right)_{3}$ (LAGP) solid electrolyte possesses a relatively high lithium ion conductivity of over $10^{-4} \mathrm{~S} / \mathrm{cm}$, high atmospheric stability, and

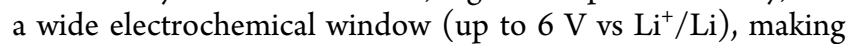
it a suitable candidate to optimize the polymer electrolyte. ${ }^{43}$ With this rationale, an HSE composed of PEO, SN, LAGP, and lithium salt (LiTFSI) was fabricated and characterized, as shown in Figure 1. The temperature dependence of ionic conductivities for the PEO-SN-LiTFSI electrolyte (SPE) and the LAGP-PEO-SN-LiTFSI electrolyte (HSE) are presented in Figure 1a. The conductivity of both electrolytes follows an Arrhenius behavior, resulting in activation energies of 0.384 and $0.381 \mathrm{eV}$ for the SPE and HSE, respectively. This indicates that the diffusion barrier is slightly smaller in the HSE and much smaller as compared to, for instance, that in the hybrid PEO-LLZO electrolyte $(0.83 \mathrm{eV}) .^{40}$ Moreover, the room-temperature conductivity of the HSE $\left(1.73 \times 10^{-4} \mathrm{~S} / \mathrm{cm}\right.$ at $\left.25^{\circ} \mathrm{C}\right)$ is 2 times higher than that of the SPE mixture $(7.58$ $\times 10^{-5} \mathrm{~S} / \mathrm{cm}$ at $25{ }^{\circ} \mathrm{C}$ ), suggesting that the LAGP filler enhances the ionic conductivity of the HSE mixture. Figure $1 \mathrm{~b}$ shows the ${ }^{7} \mathrm{Li}$ spin-lattice relaxation (SLR) rate $\left(1 / T_{1}\right)$ as a function of temperature for the HSE. The maximum relaxation rate is achieved at $338 \mathrm{~K}$, as shown in Figure $1 \mathrm{~b}$, which implies that the hopping frequency is of the order of the Larmor frequency $\left(\omega_{o}\right)$. The low-temperature flank represents the short-range motional processes, here possibly corresponding to Li-ion transport within the polymer chains, whereas the hightemperature flank most likely represents a longer-range Li-ion diffusion process, most likely corresponding to $\mathrm{Li}$-ion transport between polymer chains or transport from the polymer matrix to LAGP particles. ${ }^{44,45}$ Assuming an Arrhenius behavior for the Li-ion residence time, the SLR rates in Figure $1 \mathrm{~b}$ yield activation energies of 0.163 and $0.204 \mathrm{eV}$, respectively, for the local Li-ion and long-range Li-ion diffusion processes. SEM images of the HSE are shown in Figure 1c,d. The morphology of the surface demonstrates that a relatively flat film forms, which is free-standing and translucent, as observed in the inset of Figure 1c. According to the density of each material, the volumetric ratio of the microsized LAGP is $2.3 \%$. Figure $1 \mathrm{~d}$ shows that the LAGP particles, having an average diameter of $\sim 1 \mu \mathrm{m}$, are evenly distributed throughout the polymer matrix. Most of the LAGP particles are wrapped in the polymer electrolyte, and only a small number of particles are visible on the surface. Summarizing, it is demonstrated that introducing the LAGP in the SPE mixture results in a more processable free-standing membrane with a uniform surface morphology with a higher ionic conductivity.

The HSE was first tested electrochemically in a Li-metal symmetric cell $(\mathrm{Li} / \mathrm{HSE} / \mathrm{Li})$ and as a $\mathrm{LIB}$ combining a $\mathrm{Li}$ metal anode and $\mathrm{LiFePO}_{4}$ (LFP) cathode ( $\left.\mathrm{Li} / \mathrm{HSE} / \mathrm{LFP}\right)$, as shown in Figure 2. Without LAGP, as shown in Figure 2a, the 
(a)

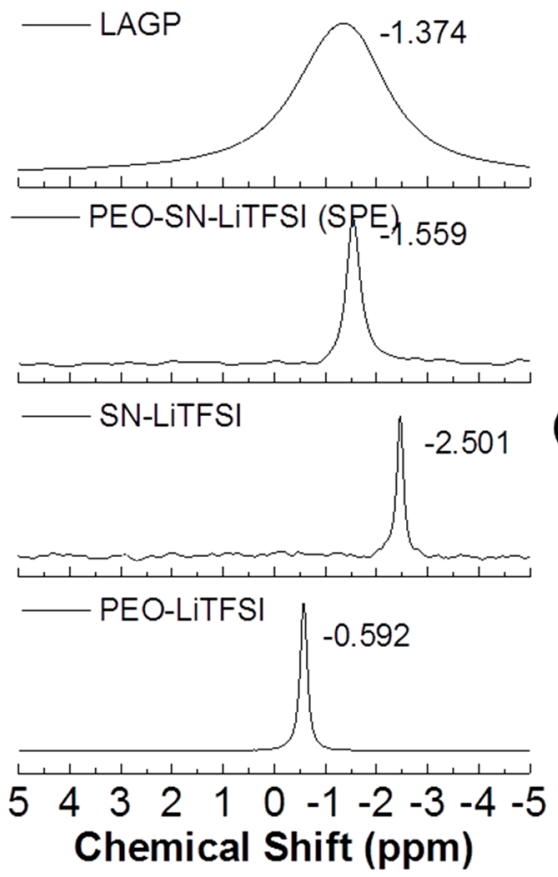

(b)

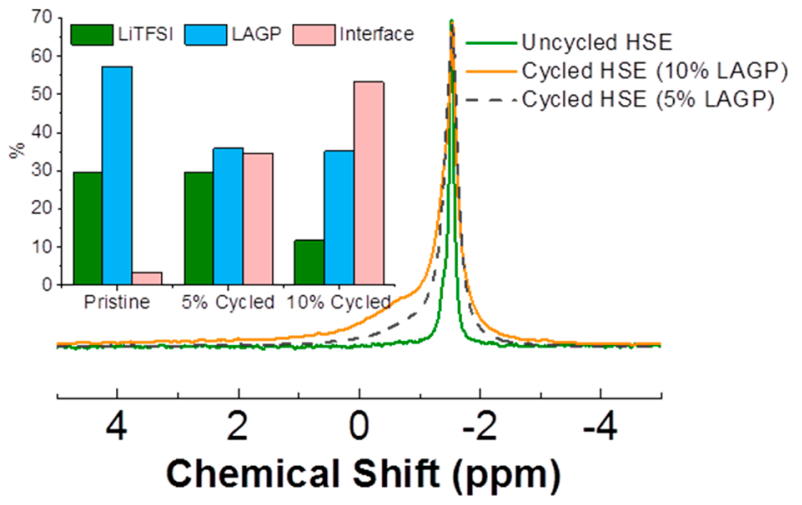

(c)

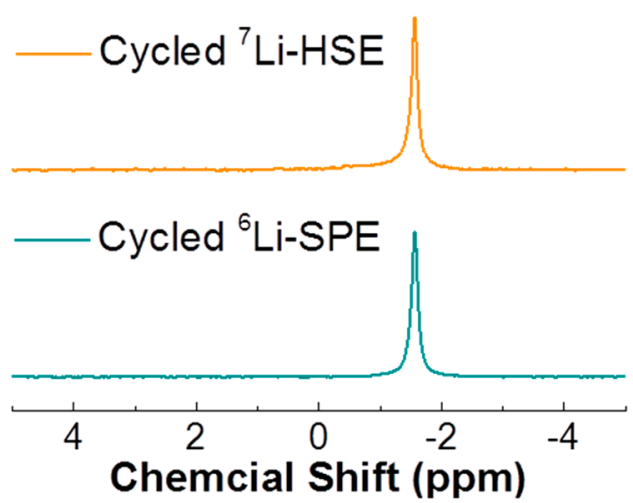

Figure 3. ssNMR characterization of the pathway of Li-ion transport in the HSE. (a) One-dimensional (1D) ${ }^{6} \mathrm{Li}$ MAS spectra of PEOLiTFSI, SN-LiTFSI, SPE, and LAGP. (b) $1 \mathrm{D}^{6} \mathrm{Li}$ MAS spectra of the HSE and HSE cycled between ${ }^{6} \mathrm{Li}$ metal and their quantification results. (c) $1 \mathrm{D}^{6} \mathrm{Li}$ MAS spectra of the SPE cycled between ${ }^{6} \mathrm{Li}$ metal and HSE cycled between ${ }^{7} \mathrm{Li}$ metal.

$\mathrm{Li} / \mathrm{SPE} / \mathrm{Li}$ cell shows an overpotential of over $200 \mathrm{mV}$ and quickly dies in less than seven cycles by a short circuit. This indicates poor resistance against dendrite formation and penetration. Upon LAGP addition, the Li/HSE/Li cell shows more stable cycling at a relatively small overpotential of $\sim 100$ $\mathrm{mV}$. The charge and discharge curves for 10 cycles of a $\mathrm{Li} /$ HSE/LFP battery, working at $0.05 \mathrm{C}$ at room temperature, are shown in Figure $2 \mathrm{~b}$. The charge and discharge curves show the expected potential plateaus of the LFP redox, indicating a reversible cycling process. ${ }^{46}$ The battery provides a capacity of $\sim 120 \mathrm{mAh} / \mathrm{g}$, with an average Coulombic efficiency of $\sim 99.9 \%$ and an over potential of $300 \mathrm{mV}$, indicating the feasibility of the HSE to serve as a solid-state electrolyte for a roomtemperature LMB. For comparison, the Li/SPE/LFP battery shows a lower capacity $(\sim 85 \mathrm{mAh} / \mathrm{g})$, a larger and growing overpotential $(\sim 500 \mathrm{mV})$, and a lower Coulombic efficiency $(\sim 97 \%)$, reflecting the low ionic conductivity and poor mechanical strength of the SPE (Figure S1). The electrochemical stability of both the SPE and HSE were investigated by linear-sweep voltammetry (LSV), as shown in Figure 2c. From the LCV scans, the HSE demonstrates a larger electrochemical stability window, up to $4.5 \mathrm{~V}$, while for the SPE, oxidation sets in at around $4.2 \mathrm{~V}$. This suggests that LAGP particles interact with PEO and the lithium salt anions, raising the oxidative stability. ${ }^{47}$ The interface stability of $\mathrm{Li} /$ $\mathrm{HSE} / \mathrm{Li}$ was further examined by electrochemical impedance spectroscopy (EIS), as shown in Figure 2d. It can be seen that the interfacial resistance of the $\mathrm{Li} / \mathrm{HSE} / \mathrm{Li}$ increases to reach the maximum value after five cycles, presumably due to the volumetric changes upon lithium-metal plating and stripping and the evolution of the lithium-HSE interface. ${ }^{48}$ After 5 cycles, the resistance continuously decreases until cycle 25, after which it remains constant up until 100 cycles, which indicates a more conductive interface forming during the initial cycles. Figure $2 \mathrm{e}$ shows the temperature-dependent ${ }^{7} \mathrm{Li} \mathrm{NMR}$ SLR rates $\left(1 / T_{1}\right)$ for both the pristine HSE and for the HSE after 10 cycles in an $\mathrm{Li} / \mathrm{HSE} / \mathrm{Li}$ cell. The temperature corresponding to the maximum SLR rate and the activation energies from the low- and high-temperature flanks (representing local and long-range diffusion) remain the same before and after cycling $(338 \mathrm{~K})$, which demonstrates that the bulk selfdiffusion of the HSE is not affected by cycling, again demonstrating the high stability of the HSE.

To understand the improved conductivity observed upon the addition of a relatively small amount of LAGP (10 wt \%), the Li-ion transport pathway was investigated. We employed ssNMR, which is demonstrated to be a powerful technique to locate $\mathrm{Li}$ ions and trace their migration and diffusion pathways in solid electrolytes. ${ }^{42,49-51}$ Figure 3 a depicts the ${ }^{6} \mathrm{Li}$ magic angle spinning (MAS) NMR spectra of PEO-LiTFSI, SNLiTFSI, SPE, and LAGP. It can be seen that the SPE shows a resonance at $-1.56 \mathrm{ppm}$, located between that observed for pristine PEO-LiTFSI $(-0.59 \mathrm{ppm})$ and SN-LiTFSI $(-2.50$ $\mathrm{ppm})$. This indicates that $\mathrm{Li}$ experiences a homogeneous environment, most likely reflecting that $\mathrm{SN}$ and PEO mix well and that the diffusion of $\mathrm{Li}$ leads to a well-defined average chemical shift, as observed. LAGP shows a relatively broad resonance peak at $-1.37 \mathrm{ppm}$, quite close to that observed for the SN-PEO-LiTFSI mixture (-1.56 ppm), making it difficult to spectrally resolve the individual LAGP and polymer electrolyte peaks in the HSE system. Nevertheless, the spectrum of the pristine HSE (Figure $3 \mathrm{~b}$ ) can be deconvoluted 
into two resonances, one representing $\mathrm{Li}$ in the LAGP and the other $\mathrm{Li}$ in SN-PEO-LiTFSI, as shown in the Supporting Information Figure S2. Next we investigated the impact of cycling the HSE (with a natural abundance of Li isotopes of $7.5 \%{ }^{6} \mathrm{Li}$ and $\left.92.5 \%{ }^{7} \mathrm{Li}\right)$ in a Li-metal symmetric cell comprising ${ }^{6} \mathrm{Li}$-enriched Li-metal electrodes. Deconvolution of the ${ }^{6} \mathrm{Li}$ NMR spectrum of the cycled HSE (Figure S2), the results of which are shown in Figure $3 b$, demonstrates that after 10 cycles a larger fraction of the ${ }^{6} \mathrm{Li}$ signal originates from LAGP as compared to the pristine HSE. This indicates that when the HSE is cycled between ${ }^{6} \mathrm{Li}$-enriched Li-metal, which leads to ${ }^{6} \mathrm{Li}$-ion migration through the HSE, results in replacement of a significant amount of ${ }^{7} \mathrm{Li}$ in the LAGP by ${ }^{6} \mathrm{Li}$, attesting that LAGP forms part of the Li-ion transport pathway. In addition, a new broad ${ }^{6} \mathrm{Li}$ resonance at $-0.75 \mathrm{ppm}$ appears upon cycling. As shown in Figure $3 b$, when the content of LAGP in the HSE is decreased from 10 to $5 \mathrm{wt} \%$ and cycled in a ${ }^{6} \mathrm{Li}$-enriched $\mathrm{Li}$-metal symmetric cell, the contribution of this new ${ }^{6} \mathrm{Li}$ resonance reduces by nearly $40 \%$. This implies that a new $\mathrm{Li}$ environment is created upon cycling. Because the amount of this environment appears to scale with the LAGP content, we propose that it arises from $\mathrm{Li}$ ions at the interface between the LAGP particles and the polymer matrix. To support this hypothesis, we also cycled the HSE between regular Li metal (comprising $\sim 7.5 \%{ }^{6} \mathrm{Li}$ ) and the SPE mixture (without LAGP) between the ${ }^{6} \mathrm{Li}$-enriched Li-metal, each for 10 cycles. As shown in Figure 3c, the cycled SPE does not display the ${ }^{6} \mathrm{Li}$ resonance at $-0.75 \mathrm{ppm}$, indicating that the new environment arises due to the presence of LAGP. In addition, the appearance of the low-intensity ${ }^{6} \mathrm{Li}$ peak at -0.75 ppm for the HSE cycled between natural abundance Li-metal further confirms that this environment is correlated with the presence of LAGP. More direct proof that the environment at $-0.75 \mathrm{ppm}$ can be assigned to the interface between LAGP and polymer components in the HSE is provided by ${ }^{1} \mathrm{H}-{ }^{6} \mathrm{Li}$ cross-polarization (CP) MAS measurements. In these experiments, transfer of polarization occurs from protons $\left({ }^{1} \mathrm{H}\right)$, in this case present in the polymer, to any ${ }^{6} \mathrm{Li}$ environment in its close vicinity. This happens during a varying time interval called the contact time, typically in the range of $100 \mu \mathrm{s}-10 \mathrm{~ms}$. (Figure S2). For both the pristine HSE and that cycled between enriched ${ }^{6} \mathrm{LiFePO}_{4}$ and $\mathrm{Li}_{4} \mathrm{Ti}_{5} \mathrm{O}_{12}$, the ${ }^{1} \mathrm{H}-{ }^{6} \mathrm{Li} \mathrm{CP}$ MAS NMR spectra reveal only the $\mathrm{Li}$ environment at -0.75 ppm, which increases in intensity as the contact time increases, as shown in the Supporting Information Figure S3a,b. This process depends on the heteronuclear dipolar coupling between ${ }^{1} \mathrm{H}$ and ${ }^{6} \mathrm{Li}$ and thus is most efficient when these species are in close proximity to each other. This indicates that the observed ${ }^{6} \mathrm{Li}$ environment is in close spatial proximity to the polymer phase of the HSE. In addition, as it only appears in the presence of the LAGP phase, we conclude that it must represent an environment close to the interface between the LAGP and the polymer phase in the HSE. Interesting, the CP intensity builds up less strongly for the cycled HSE (Supporting Information Figure S3c), which may indicate that locally the ${ }^{6} \mathrm{Li}$ ions are more mobile upon cycling, which weakens the ${ }^{1} \mathrm{H}-{ }^{6} \mathrm{Li}$ dipolar interaction, making the process less efficient. On the basis of the ${ }^{6} \mathrm{Li}$ NMR analysis, we propose that the Li-ion transport through the HSE for a significant part takes place through the LAGP particles, as well as through the interface environment located between the LAGP particles and polymer matrix of the HSE.
Figure 4 schematically shows the pathway of Li-ion transport as proposed for previous $\mathrm{HSEs}^{30,34,39,40}$ and found for the (a)

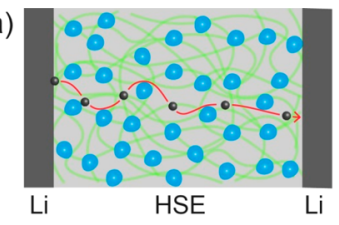

(c)

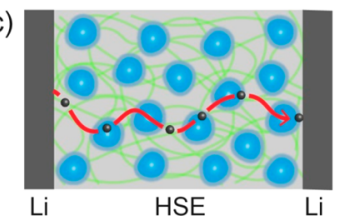

(b)
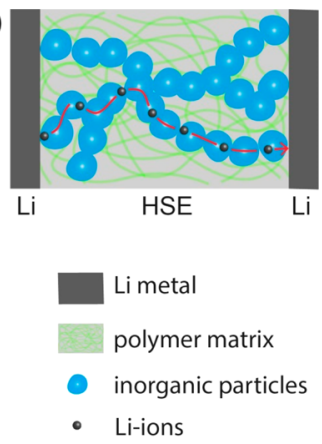

Figure 4. Schematic representation of the Li-ion diffusion pathways in PEO-based HSEs with (a) a small amount of nanofillers, $^{30,39}$ (b) a large fraction of solid electrolyte microfillers, ${ }^{34,40}$ and (c) a small fraction of micro-LAGP in the present LAGP-SN-PEO HSE.

present HSE. Shown in Figure 4a, nanosized inorganic fillers have been shown to suppresses the crystallization of PEO, thereby enhancing the polymer segmental motion for ionic conduction near room temperature. In this case, Li-ion transport takes place along the polymer chain, near the inorganic filler surface; hence, the latter is inactive with respect to the Li-ion transport. ${ }^{30,39}$ This strategy results in ionic conductivities up to $\sim 10^{-5} \mathrm{~S} \mathrm{~cm}^{-1}$ at $30^{\circ} \mathrm{C}$, which is not sufficient for room-temperature battery operation. Shown in Figure $4 \mathrm{~b}$, in an HSE with a large amount of microsized inorganic filler ( $50 \mathrm{wt} \%)$, Li-ion transport takes place through the solid electrolyte filler, avoiding the polymer phase. In this case, the solid electrolyte should form a percolating pathway, ${ }^{52,53}$ which requires a large volume fraction of filler, and the polymer phase is considered inactive with respect to the conduction mechanism. The transport mechanism proposed here is fundamentally different from previously demonstrated mechanisms in HSE as it involves conductivity through the LAGP particles, the polymer phase, and the interface between these two phases. As shown in Figure 4c, we propose that the interface environment between the SN-PEO-LiTFSI and the LAGP facilitates the exchange, activating the conductivity through the LAGP. This synergy is responsible for the high ionic conductivity of $1.6 \times 10^{-4} \mathrm{~S} / \mathrm{cm}$ obtained, even with the relatively small fraction of LAGP present, and the resulting low overpotential of $100 \mathrm{mV}$ for a Li-metal symmetric cell.

To conclude, we have prepared an HSE comprising PEO, $\mathrm{SN}$, and Li salt as the polymer matrix and up to $10 \mathrm{wt} \%$ LAGP as the inorganic filler. The processable and flexible solid electrolyte provides a Li-ion conductivity of $1.73 \times 10^{-4} \mathrm{~S} / \mathrm{cm}$, achieved with an advantageously small fraction of inorganic solid electrolyte. The HSE demonstrates promising, stable, room-temperature cycling of a LMB, utilizing LFP as the cathode. Detailed investigation of the transport mechanism of the HSE with ssNMR proves that LAGP contributes to the conductivity, enabled by an interface with the polymer matrix that allows facile exchange. This rationalizes the high Li-ion conductivity and represents a fundamentally different transport mechanism compared to known HSEs, where both phases participate in the Li-ion transport. This in-tandem conductivity concept appears to be a promising strategy toward the challenges posed by solid-state LMBs and hence toward 
improving both the energy density and safety of reversible energy storage in batteries.

\section{ASSOCIATED CONTENT}

\section{S Supporting Information}

The Supporting Information is available free of charge on the ACS Publications website at DOI: 10.1021/acsenergylett.9b01371.

${ }^{6} \mathrm{Li}$ MAS NMR spectra and spectral deconvolution for the HSE, ${ }^{1} \mathrm{H}-{ }^{6} \mathrm{Li} \mathrm{CP}$ spectra as a function of contact times for the HSE, and a detailed experimental section (PDF)

\section{AUTHOR INFORMATION}

\section{Corresponding Author}

*E-mail: M.Wagemaker@tudelft.nl.

\section{ORCID}

Marnix Wagemaker: 0000-0003-3851-1044

\section{Author Contributions}

M.L. and Z.C. contributed equally to this work.

\section{Notes}

The authors declare no competing financial interest.

\section{ACKNOWLEDGMENTS}

The authors thank Michel Steenvoorden and Frans Ooms for their assistance with experiments. M.L. acknowledges support by The Netherlands Organization for Scientific Research (NWO) under Grant No. 15788 and M.W. acknowledges support under VICI Grant No. 16122. Financial support from the Advanced Dutch Energy Materials (ADEM) program of the Dutch Ministry of Economic Affairs, Agriculture and Innovation is gratefully acknowledged. We would like to thank the Chinese Scholarship Council (CSC) for financially supporting part of the work in this paper.

\section{REFERENCES}

(1) Xu, W.; Wang, J.; Ding, F.; Chen, X.; Nasybulin, E.; Zhang, Y.; Zhang, J. Lithium metal anodes for rechargeable batteries. Energy Environ. Sci. 2014, 7, 513.

(2) Wood, K. N.; Noked, M.; Dasgupta, N. P. Lithium metal anodes: toward an improved understanding of coupled morphological, electrochemical, and mechanical behavior. ACS Energy Letters 2017, 2, 664 .

(3) Cheng, X.; Zhang, R.; Zhao, C.; Zhang, Q. Toward Safe Lithium Metal Anode in Rechargeable Batteries: A Review. Chem. Rev. 2017, 117, 10403-10473.

(4) Lin, D.; Liu, Y.; Cui, Y. Reviving the lithium metal anode for high-energy batteries. Nat. Nanotechnol. 2017, 12, 194.

(5) Yang, Y.; Zheng, G.; Cui, Y. Nanostructured sulfur cathodes. Chem. Soc. Rev. 2013, 42, 3018.

(6) Luntz, A. C.; McCloskey, B. D. Nonaqueous Li-air batteries: a status report. Chem. Rev. 2014, 114, 11721.

(7) Manthiram, A.; Fu, Y.; Chung, S.; Zu, C.; Su, Y. Rechargeable lithium-sulfur batteries. Chem. Rev. 2014, 114, 11751.

(8) Liu, M.; Qin, X.; He, Y.; Li, B.; Kang, F. Recent innovative configurations in high-energy lithium-sulfur batteries. J. Mater. Chem. A 2017, 5, 5222.

(9) Aurbach, D.; Zinigrad, E.; Cohen, Y.; Teller, H. A short review of failure mechanisms of lithium metal and lithiated graphite anodes in liquid electrolyte solutions. Solid State Ionics 2002, 148, 405.

(10) Zhang, T.; Liao, K.; He, P.; Zhou, H. A self-defense redox mediator for efficient lithium- $\mathrm{O}_{2}$ batteries. Energy Environ. Sci. 2016, 9, 1024.
(11) Liu, Y.; Lin, D.; Li, Y.; Chen, G.; Pei, A.; Nix, O.; Li, Y.; Cui, Y. Solubility-mediated sustained release enabling nitrate additive in carbonate electrolytes for stable lithium metal anode. Nat. Commun. 2018, 9, 3656.

(12) Zheng, J.; Engelhard, M. H.; Mei, D.; Jiao, S.; Polzin, B. J.; Zhang, J.; Xu, W. Electrolyte additive enabled fast charging and stable cycling lithium metal batteries. Nature Energy 2017, 2, 17012.

(13) Liu, Y.; Lin, D.; Liang, Z.; Zhao, J.; Yan, K.; Cui, Y. Lithiumcoated polymeric matrix as a minimum volume-change and dendritefree lithium metal anode. Nat. Commun. 2016, 7, 10992.

(14) Liu, Y.; Lin, D.; Yuen, P. Y.; Liu, K.; Xie, J.; Dauskardt, R. H.; Cui, Y. An artificial solid electrolyte interphase with high Li-ion conductivity, mechanical strength, and flexibility for stable lithium metal anodes. Adv. Mater. 2017, 29, 1605531.

(15) Yan, K.; Lu, Z.; Lee, H.; Xiong, F.; Hsu, P.; Li, Y.; Zhao, J.; Chu, S.; Cui, Y. Selective deposition and stable encapsulation of lithium through heterogeneous seeded growth. Nature Energy 2016, 1, 16010 .

(16) Yun, Q.; He, Y.; Lv, W.; Zhao, Y.; Li, B.; Kang, F.; Yang, Q. Chemical dealloying derived 3D porous current collector for Li metal anodes. Adv. Mater. 2016, 28, 6932.

(17) Zhang, C.; Lv, W.; Zhou, G.; Huang, Z.; Zhang, Y.; Lyu, R.; Wu, H.; Yun, Q.; Kang, F.; Yang, Q. Vertically aligned lithiophilic $\mathrm{CuO}$ nanosheets on a $\mathrm{Cu}$ collector to stabilize lithium deposition for lithium metal batteries. Adv. Energy Mater. 2018, 8, 1703404.

(18) Liang, Z.; Lin, D.; Zhao, J.; Lu, Z.; Liu, Y.; Liu, C.; Lu, Y.; Wang, H.; Yan, K.; Tao, X.; Cui, Y. Composite lithium metal anode by melt infusion of lithium into a 3D conducting scaffold with lithiophilic coating. Proc. Natl. Acad. Sci. U. S. A. 2016, 113, 2862.

(19) Zeng, X.; Yin, Y.; Li, N.; Du, W.; Guo, Y.; Wan, L. Reshaping lithium plating/stripping behavior via bifunctional polymer electrolyte for room-temperature solid Li metal batteries. J. Am. Chem. Soc. 2016, 138,15825 .

(20) Zhou, W.; Wang, S.; Li, Y.; Xin, S.; Manthiram, A.; Goodenough, J. B. Plating a dendrite-free lithium anode with a polymer/ceramic/polymer sandwich electrolyte. J. Am. Chem. Soc. 2016, 138, 9385.

(21) Khurana, R.; Schaefer, J. L.; Archer, L. A.; Coates, G. W. Suppression of lithium dendrite growth using cross-linked polyethylene/poly(ethylene oxide) electrolytes: A new approach for practical lithium-metal polymer batteries. J. Am. Chem. Soc. 2014, 136, 7395.

(22) Janek, J.; Zeier, W. G. A solid future for battery development. Nature Energy 2016, 1, 16141.

(23) $\mathrm{Xu}, \mathrm{K}$. Electrolytes and interphases in Li-ion batteries and beyond. Chem. Rev. 2014, 114, 11503.

(24) $\mathrm{Xu}, \mathrm{K}$. Nonaqueous liquid electrolytes for lithium-based rechargeable batteries. Chem. Rev. 2004, 104, 4303.

(25) Meyer, W. H. Polymer electrolytes for lithium-ion batteries. Adv. Mater. 1998, 10, 439

(26) Varzi, A.; Raccichini, R.; Passerini, S.; Scrosati, B. Challenges and prospects of the role of solid electrolytes in the revitalization of lithium metal batteries. J. Mater. Chem. A 2016, 4, 17251.

(27) Armand, M.; Chabagno, J.; Duclot, M. Poly-ethers as solid electrolytes. Fast Ion Transport in Solids 1979, 131.

(28) Yue, L.; Ma, J.; Zhang, J.; Zhao, J.; Dong, S.; Liu, Z.; Cui, G.; Chen, L. All solid-state polymer electrolytes for high-performance lithium ion batteries. Energy Storage Materials 2016, 5, 139.

(29) Song, J.; Wang, Y.; Wan, C. C. Review of gel-type polymer electrolytes for lithium-ion batteries. J. Power Sources 1999, 77, 183.

(30) Lin, D.; Liu, W.; Liu, Y.; Lee, H. R.; Hsu, P.; Liu, K.; Cui, Y. High ionic conductivity of composite solid polymer electrolyte via in situ synthesis of monodispersed $\mathrm{SiO}_{2}$ nanospheres in poly (ethylene oxide). Nano Lett. 2016, 16, 459.

(31) Liu, W.; Liu, N.; Sun, J.; Hsu, P.; Li, Y.; Lee, H.; Cui, Y. Ionic conductivity enhancement of polymer electrolytes with ceramic nanowire fillers. Nano Lett. 2015, 15, 2740.

(32) Liu, W.; Lee, S. W.; Lin, D.; Shi, F.; Wang, S.; Sendek, A. D.; Cui, Y. Enhancing ionic conductivity in composite polymer 
electrolytes with well-aligned ceramic nanowires. Nature Energy 2017, 2, 17035 .

(33) Zheng, J.; Hu, Y. New Insights into the Compositional Dependence of Li-Ion Transport in Polymer-Ceramic Composite Electrolytes. ACS Appl. Mater. Interfaces 2018, 10, 4113.

(34) Zheng, J.; Tang, M.; Hu, Y. Lithium Ion Pathway within $\mathrm{Li}_{7} \mathrm{La}_{3} \mathrm{Zr}_{2} \mathrm{O}_{12}$-Polyethylene Oxide Composite Electrolytes. Angew. Chem., Int. Ed. 2016, 55, 12538.

(35) Yang, T.; Zheng, J.; Cheng, Q.; Hu, Y.; Chan, C. K. Composite polymer electrolytes with $\mathrm{Li}_{7} \mathrm{La}_{3} \mathrm{Zr}_{2} \mathrm{O}_{12}$ garnet-type nanowires as ceramic fillers: mechanism of conductivity enhancement and role of doping and morphology. ACS Appl. Mater. Interfaces 2017, 9, 21773. (36) Wang, W.; Yi, E.; Fici, A. J.; Laine, R. M.; Kieffer, J. Lithium ion conducting poly (ethylene oxide)-based solid electrolytes containing active or passive ceramic nanoparticles. J. Phys. Chem. C 2017, 121, 2563.

(37) Liu, W.; Lin, D.; Sun, J.; Zhou, G.; Cui, Y. Improved lithium ionic conductivity in composite polymer electrolytes with oxide-ion conducting nanowires. ACS Nano 2016, 10, 11407.

(38) Kim, M.; Lee, L.; Jung, Y.; Kim, S. Study on Ion Conductivity and Crystallinity of Composite Polymer Electrolytes Based on Poly (ethylene oxide)/Poly (acrylonitrile) Containing Nano-Sized $\mathrm{Al}_{2} \mathrm{O}_{3}$ Fillers. J. Nanosci. Nanotechnol. 2013, 13, 7865.

(39) Zheng, J.; Wang, P.; Liu, H.; Hu, Y. Interface-Enabled Ion Conduction in $\mathrm{Li}_{10} \mathrm{GeP}_{2} \mathrm{~S}_{12}$-Polyethylene Oxide Hybrid Electrolytes. ACS Applied Energy Materials 2019, 2, 1452-1459.

(40) Zhao, C.; Zhang, X.; Cheng, X.; Zhang, R.; Xu, R.; Chen, P.; Peng, H.; Huang, J.; Zhang, Q. An anion-immobilized composite electrolyte for dendrite-free lithium metal anodes. Proc. Natl. Acad. Sci. U. S. A. 2017, 114, 11069-11074.

(41) Fan, L.; Hu, Y.; Bhattacharyya, A. J.; Maier, J. Succinonitrile as a versatile additive for polymer electrolytes. Adv. Funct. Mater. 2007, 17, 2800.

(42) Yu, C.; Ganapathy, S.; de Klerk, N. J.; Roslon, I.; van Eck, E. R.; Kentgens, A. P.; Wagemaker, M. Unravelling Li-ion transport from picoseconds to seconds: bulk versus interfaces in an argyrodite $\mathrm{Li}_{6} \mathrm{PS}_{5} \mathrm{Cl}-\mathrm{Li}_{2} \mathrm{~S}$ all-solid-state Li-Ion battery. J. Am. Chem. Soc. 2016, $138,11192$.

(43) Yi, J.; Liu, Y.; Qiao, Y.; He, P.; Zhou, H. Boosting the cycle life of $\mathrm{Li}-\mathrm{O}_{2}$ batteries at elevated temperature by employing a hybrid polymer-ceramic solid electrolyte. ACS Energy Letters 2017, 2, 1378.

(44) Yu, C.; Ganapathy, S.; De Klerk, N. J. J.; Roslon, I.; Van Eck, E. R. H.; Kentgens, A. P. M.; Wagemaker, M. Unravelling Li-Ion Transport from Picoseconds to Seconds: Bulk versus Interfaces in an Argyrodite $\mathrm{Li}_{6} \mathrm{PS}_{5} \mathrm{Cl}_{-} \mathrm{Li}_{2} \mathrm{~S}$ All-Solid-State Li-Ion Battery. J. Am. Chem. Soc. 2016, 138, 11192.

(45) Epp, V.; Wilkening, M. Li-ion dynamics in solids as seen via relaxation NMR.Materials and Energy; 2015; Vol. 6, p 133.

(46) Yuan, L.; Wang, Z.; Zhang, W.; Hu, X.; Chen, J.; Huang, Y.; Goodenough, J. B. Development and challenges of $\mathrm{LiFePO}_{4}$ cathode material for lithium-ion batteries. Energy Environ. Sci. 2011, 4, 269.

(47) Zhao, Y.; Huang, Z.; Chen, S.; Chen, B.; Yang, J.; Zhang, Q.; Ding, F.; Chen, Y.; Xu, X. A promising PEO/LAGP hybrid electrolyte prepared by a simple method for all-solid-state lithium batteries. Solid State Ionics 2016, 295, 65.

(48) Feng, W.; Dong, X.; Lai, Z.; Zhang, X.; Wang, Y.; Wang, C.; Luo, J.; Xia, Y. Building an Interfacial Framework: Li/Garnet Interface Stabilization Through a $\mathrm{Cu}_{6} \mathrm{Sn}_{5}$ Layer. ACS Energy Letters 2019, 4, 1725-1731.

(49) Ganapathy, S.; Yu, C.; van Eck, E. R.; Wagemaker, M. Peeking Across Grain Boundaries in a Solid-State Ionic Conductor. ACS Energy Letters 2019, 4, 1092-1097.

(50) Yu, C.; Ganapathy, S.; Van Eck, E. R.; Wang, H.; Basak, S.; Li, Z.; Wagemaker, M. Accessing the bottleneck in all-solid state batteries, lithium-ion transport over the solid-electrolyte-electrode interface. Nat. Commun. 2017, 8, 1086.

(51) Kuhn, A.; Kunze, M.; Sreeraj, P.; Wiemhöfer, H.; Thangadurai, V.; Wilkening, M.; Heitjans, P. NMR relaxometry as a versatile tool to study Li ion dynamics in potential battery materials. Solid State Nucl. Magn. Reson. 2012, 42, 2.

(52) Yamada, H.; Bhattacharyya, A. J.; Maier, J. Extremely high silver ionic conductivity in composites of silver halide $(\mathrm{AgBr}, \mathrm{AgI})$ and mesoporous alumina. Adv. Funct. Mater. 2006, 16, 525.

(53) Chen, L.; Li, Y.; Li, S.; Fan, L.; Nan, C.; Goodenough, J. B. $\mathrm{PEO} /$ garnet composite electrolytes for solid-state lithium batteries: from "ceramic-in-polymer" to "polymer-in-ceramic. Nano Energy 2018, 46, 176. 